\title{
GROUND MOTION AND VIBRATION ISSUES FOR ACCELERATORS*
}

\author{
Andrei Seryi, SLAC, Stanford, USA
}

\begin{abstract}
Ground motion and vibration can be a limiting factor in the performance of future colliders, in particular linear colliders and large hadron colliders. Investigations of ground motion have been carried out around the world for several decades. In this review, after brief discussion of ground motion requirements for NLC, TESLA and VLHC, the results of recent investigations of ground motion are presented as well as stabilization techniques developed for the NLC.
\end{abstract}

\section{INTRODUCTION}

New accelerators to meet the demands of particle physics experiments will have to push both the energy and luminosity frontiers. The technical requirements for these machines are beyond what has typically been achieved. The major candidates for a next generation machine are electron-positron colliders, NLC [1,2], JLC [3] and TESLA [4] optimized to study the $0.2-1.5 \mathrm{TeV}$ energy range, and also a hadron collider VLHC [5] and electronpositron collider CLIC [6] that would probe multi-TeV physics. Ground motion and vibration are of concern for all these machines, but the details of the problems are different. These differences are reflected in the level of detail given to the design of systems to stabilize the beam and the luminosity.

Ground motion and vibration have two important effects on a collider. They can cause the beams to miss each other at the interaction point (IP), and they can cause beam emittance growth, which reduces the luminosity. In hadron colliders, such as VLHC, the beam size is still sufficiently large (smallest size $250 \mathrm{~nm}$ ) compared to the ground motion amplitude at relevant frequencies, that the offset of the beams at the IP is not an issue (all the numbers are for the high field VLHC with $87.5 \mathrm{TeV}$ beam [5]). However, the effects of ground motion can accumulate and result in emittance growth. In the VLHC, the primary issue is that movement of the quadrupoles induces betatron oscillations, which then decohere and turn into emittance growth. The lowest frequency of ground motion contributing to this effect is $\mathrm{f}=\Delta \mathrm{V} * \mathrm{f}_{0}$ where $\mathrm{f}_{0}$ is the revolution frequency $(\sim 1.3 \mathrm{kHz})$ and $\Delta v$ is the fractional tune $(\sim 0.18)$, i.e. the lowest relevant frequency is $\mathrm{f} \sim 250 \mathrm{~Hz}$. The emittance growth rate from white noise quadrupole motion without any feedback is given by $\mathrm{d} \varepsilon_{\mathrm{n}} / \mathrm{dt} \approx \mathrm{f}_{0} \gamma\langle\beta\rangle \mathrm{N}(\sigma / \mathrm{F})^{2} / 2[7,8]$ where $\mathrm{N}=1700$ is the

*Work supported by the U.S. Department of Energy, Contract DE-AC03-76SF00515 number of quads, $\mathrm{F}=100 \mathrm{~m}$ is their focal length, $<\beta>=230 \mathrm{~m}$ is the average beta function, $\gamma=9.3 \mathrm{E} 4$ is a relativistic factor and $\sigma$ is the rms vibration of quadrupoles. For these parameters, the initial emittance of $\varepsilon_{\mathrm{n}}=1.5 \mathrm{~mm}$-mrad would double in 2.5 hours with only $0.3 \mathrm{~nm}$ of quad vibration (the synchrotron radiation damping time is 2.5 hours for VLHC). This tolerance could be eased by perhaps a factor of 10 with orbit feedback. It is worth noting that the VLHC will require orbit feedbacks, not primarily because of vibrations and ground motion, but because of TMCI, resistive wall and other instabilities that may grow over several turns. Suppression of these instabilities will require sophisticated feedback systems to be developed and tested [9]. Assuming that proper feedbacks to suppress instabilities are developed, one can conclude that in a quiet location, ground motion is sufficiently small for the VLHC (see Figs.1-3). The real concern for VLHC is not the ground motion itself, but vibrations produced by equipment, for example by cryogenic systems. Design of VLHC girders and cryostats is another issue requiring attention since the amplification of ground vibrations by girder and cryostat and in-cryostat generated vibrations need to be minimized.

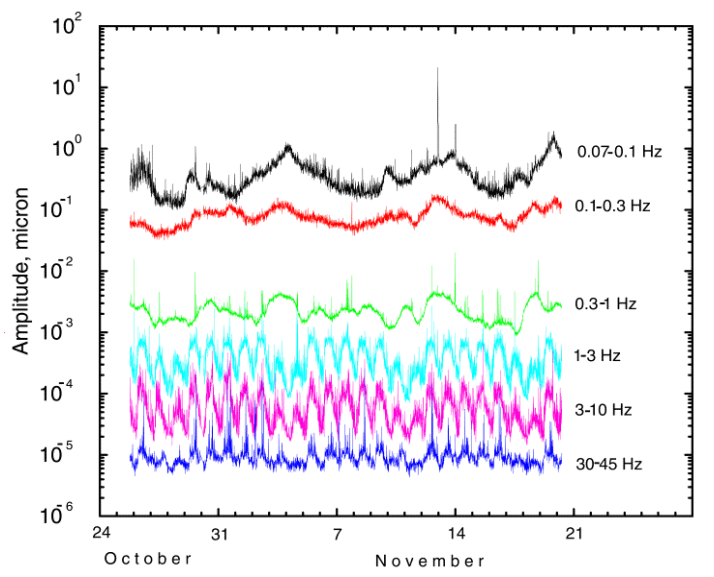

Figure 1: Ground motion measured in a typical quiet underground location: Hiidenvesi cave. RMS amplitude is shown in different frequency bands [10]. Natural ground motion is very low at high frequencies.

In current linear collider designs, the beam size at the interaction point is nanometer scale $(2-5 \mathrm{~nm})$. Ground motion can not only produce emittance growth, as for circular machines, but also cause the beams to be offset at the IP. The relevant frequencies are determined by the repetition rate of collisions $\mathrm{f}_{\text {rep }}$. The SLC experience has shown that frequencies higher than about $\mathrm{f}_{\mathrm{c}}=\mathrm{f}_{\text {rep }} / 20$ cannot be adequately corrected by a pulse-to-pulse feedback operating at the repetition rate. Therefore, it is these 
frequencies that are the source of beam offsets at the IP. Slower motion can be compensated by feedback and thus only causes beam emittance growth.

The TESLA and NLC linear collider projects differ significantly in terms of their repetition rate. The $f_{\text {rep }}$ for bunch trains at TESLA is $3-5 \mathrm{~Hz}$, so that $\mathrm{f}_{\mathrm{c}} \sim 0.2 \mathrm{~Hz}$ while NLC with a repetition rate $120 \mathrm{~Hz}$ has feedback cutoff frequency around $\mathrm{f}_{\mathrm{c}}=6 \mathrm{~Hz}$. The tolerance for uncorrelated motion of linac quadrupoles above cut-off frequency is roughly $10 \mathrm{~nm}$ for both TESLA and NLC $[2,4]$. Such motion would produce a tolerable beam offset at the IP about $0.25 \sigma_{\mathrm{y}}$ for NLC and $0.1 \boldsymbol{\sigma}_{\mathrm{y}}$ for TESLA (higher precision of collision is needed for TESLA because of higher beam-beam disruption).

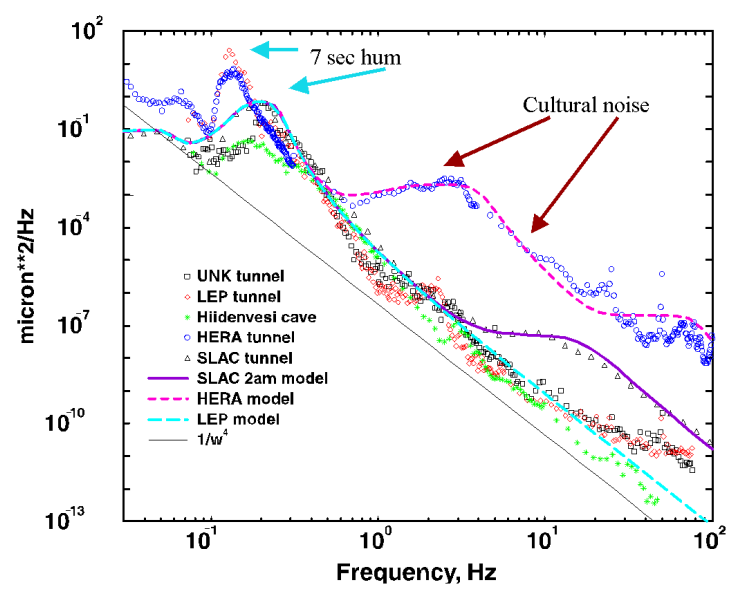

Figure 2: Power spectrum of absolute ground motion measured at different sites $[11,12]$. Smooth curves show modeling spectra. The high noise level at HERA is caused by cultural noise and, supposedly, by resonances of the clay/sandy site itself.

One can see from Fig. 3 that uncorrelated motion above $0.2 \mathrm{~Hz}$ can be about equal or several times higher than the tolerance for TESLA. Ground motion similar to that in the LEP tunnel would be acceptable, if any additional motion of the quadrupoles, which are located inside cryostats, with respect to the ground will be sufficiently small. It is therefore practically impossible for TESLA, due to its low repetition rate, to rely on the quietness of a site. Instead, for collision stabilization, TESLA must rely on fast intratrain correction [4], so that the correction would be based on $3 \mathrm{MHz}$ repetition rate of the bunch collisions. The proposed design uses the first hundred or so bunches in the train (train duration is about $1 \mathrm{msec}$ ) to correct the offset for the rest of the train. It is essential for this correction that the transverse position of the bunches in the train do not fluctuate significantly, or at least that such fluctuations are static in time, i.e. they do not fluctuate from train to train so that prediction can improve the response latency. Any phenomena that may spoil this static picture, such as poorly damped high order trapped modes in the TESLA accelerating cavities or acoustical vibrations in the tens to hundreds of $\mathrm{kHz}$ range, must be controlled.
The NLC linear collider has a high repetition rate $120 \mathrm{~Hz}$, and therefore the feedback cutoff frequency is $\mathrm{f}_{\mathrm{c}}=6 \mathrm{~Hz}$. Natural ground motion is quite low at these frequencies, as low as $0.1 \mathrm{~nm}$. Even in the SLAC linac, which is a shallow, cut-and-cover tunnel, the ground motion above $6 \mathrm{~Hz}$ is only about a nanometer. This is already stable enough for NLC. For collision stabilization, NLC can rely on the quietness of a tunnel built in favorable geology together with careful engineering control of possible vibration sources in the tunnel. In addition, the NLC quadrupoles are compact iron or permanent magnets, so it is possible to design very rigid supports that do not amplify ground motion, as was demonstrated at FFTB [13]. Moreover, the fast intratrain feedback, indispensable for TESLA, is also being developed for NLC as an additional measure for collision stabilization.

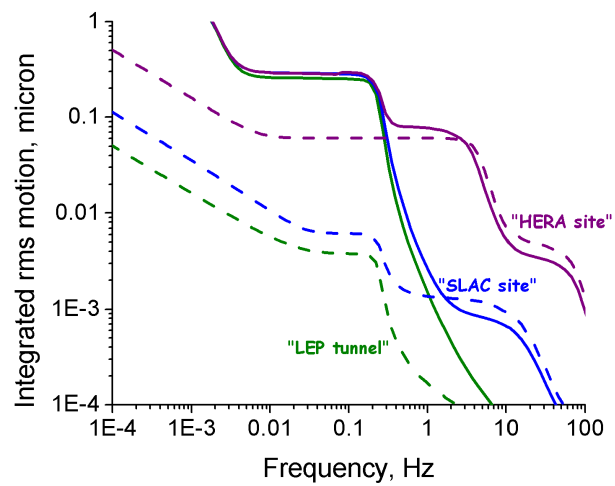

Figure 3: Example of integrated ground motion spectra based on models for "SLAC site", "HERA site" and "LEP tunnel". Absolute motion (solid curves) and differential motion for points separated by $50 \mathrm{~m}$ (dashed curves) [14].

TESLA and NLC also differ in the sensitivity of the beam emittance to quadrupole offsets caused by slow ground motion or inaccuracies of alignment. TESLA's large superconducting cavities with low wakefields and small beam energy spread make the quad alignment tolerances rather loose. For example, the misalignment of quadrupoles tolerable before a beam-based alignment would be needed, is about $25 \mu \mathrm{m}$ over $50 \mathrm{~m}$ for TESLA [4] and about $1 \mu \mathrm{m}$ over $50 \mathrm{~m}$ for NLC. Therefore, TESLA can tolerate larger diffusive ground motion and less comfortable geological conditions. Regardless of this, the girders would need to be carefully designed to minimize any additional drifts of quadrupoles with respect to ground. In the TESLA design, where the quadrupoles are located inside the cryostat, a careful engineering balance between mechanical stability and cryogenic constraints is required since usually rather thin and flexible supports are used to minimize heat loss in cryostats.

On the other hand, a quiet tunnel built in good geology, as supposed for NLC, would reduce the amount of slow motion and decrease emittance growth. As discussed below, a combination of orbit correction feedback and 
beam based alignment, similar to what was developed at SLC, will be adequate to stabilize the NLC beam emittance.

In the following sections, the ground motion studies are briefly reviewed, followed by a discussion of NLC stability in terms of ground motion.

\section{GROUND MOTION STUDIES}

Ground motion can be divided into 'fast' and 'slow', as described above. For NLC parameters, the boundary between 'fast' and 'slow' motion is in the region of a few Hz. Fast motion is typically represented by a power spectrum as shown in Fig.2. The integrated spectrum over a particular frequency band gives the corresponding RMS motion as shown in Fig.1. It is easy to see that the natural ground motion is quite small, as low as a fraction of a nanometer, for frequencies higher than several $\mathrm{Hz}$ (see Figs.1 and 3). The motion in the low frequency bands in Fig. 1 is much larger, on the order of a micrometer. However, it is important to emphasize that the amplitude shown is absolute, i.e. it is the motion of a single point with respect to an inertial reference frame. What is important for a linear collider is the relative motion of two quadrupoles separated by distances less than the betatron wavelength. Correlation measurements have shown $[1,10,15,16]$ that natural ground motion consists mostly of elastic waves with a wavelength given by the phase velocity in the media. The low frequency portion of the 'fast' motion has quite a long wavelength and is therefore highly correlated (see Fig.3). Thus, this motion is not harmful for a linear collider assuming that low frequency girder resonances do not degrade the correlation.

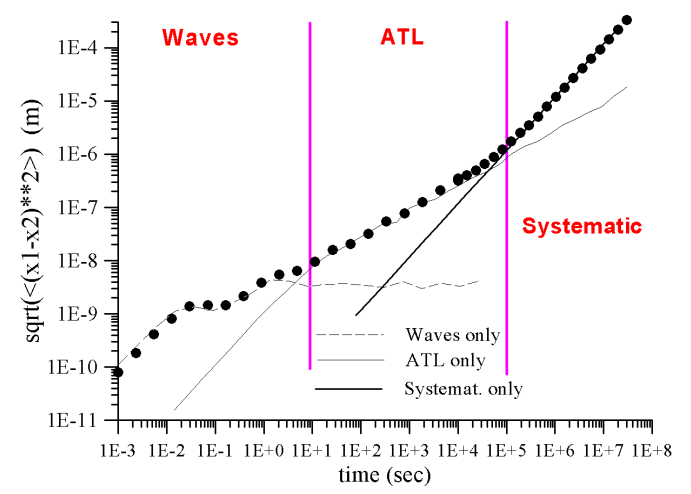

Figure 4: RMS relative motion versus time for two points separated by $30 \mathrm{~m}$ for the 2 AM SLAC site ground motion model [14].

In the NLC, the tolerance on jitter in the position of an element depends on its location. The tolerance for the Final Doublet (FD) is a fraction of the beam size at the IP, around $1 \mathrm{~nm}$. Some of the quadrupoles in the beam delivery system have tolerances roughly $5-10 \mathrm{~nm}$. The jitter tolerance for the main linac quadrupoles is $10 \mathrm{~nm}$. The relevant frequency range for these tolerances is $f_{c}>6$ Hz. For comparison, the motion of an FFTB quadrupole mounted on NLC-like movers and sitting on an Anocast girder, with water flowing in the quadrupole, differs from the floor motion by only $2 \mathrm{~nm}$ in the relevant frequency band [13]. This is sufficient for NLC requirements even though the environment was relatively noisy. Further improvements, such as slower water flow and improved girders, are also envisaged.

From these considerations, one can conclude that natural fast ground motion does not represent a limitation for NLC. The real concern is cultural noise produced in the vicinity of the accelerator, whether external or internal to the tunnel, and vibrations produced on the accelerator girder themselves. It is clear that the accelerator equipment and the NLC detector must be carefully designed with a goal of minimizing vibration. All of the conventional facilities support equipment for the accelerator will have to satisfy strict vibration criteria. This may seem to be a new or unusual constraint for accelerator designers, but is standard practice in some areas of engineering. For example, the LIGO project successfully applied various methods of passive vibration isolation to noise sources in order to achieve a sufficiently quiet environment [17].

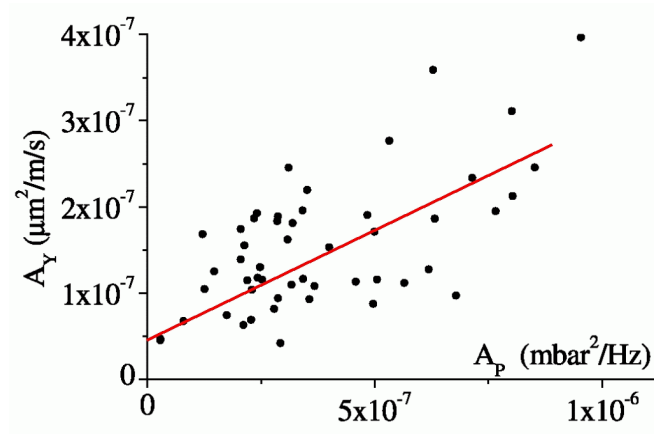

Figure 5: Parameter A of the ATL law defined from vertical motion of SLC tunnel plotted against the amplitude $A_{p}$ of the atmospheric pressure spectrum [18].

It is interesting to note that the high level of noise measured at HERA site, apparent in Fig.2, may be caused not only by cultural noises, but also by resonances of clay/sandy site itself (resonances exhibit as a minimum of vibration attenuation with distance at certain frequencies). For example, preliminary studies at two LIGO sites indicated [19] that the Livingston LIGO site, which is based on water logged clay, exhibit resonances at $1-5 \mathrm{~Hz}$, while the Hanford LIGO site built on dry sand appears to have resonances at 5-12 $\mathrm{Hz}$. This phenomenon, in particular the role of water and geology, deserves further attention.

The fast motion and vibration discussed above is not the only issue for a linear collider. Ground motion below $0.01 \mathrm{~Hz}$ or so, in spite of being very slow, can have a rather short wavelength, causing misalignments of the collider and producing emittance growth. This motion is not wave-like and can be inelastic. There are two types of motion - one is diffusive and another is systematic. The 
model for the diffusive motion parameterizes the RMS relative misalignment as an ATL law [7,20]: $\Delta X^{2}=$ ATL where $\mathrm{T}$ is the time since perfect alignment and $\mathrm{L}$ is the distance between points.

The parameter A varies by a few orders of magnitude when measured in different locations and is clearly site and geology dependent. For example, measurements at the DESY site gave a value of $\mathrm{A}=10^{-5} \mu \mathrm{m}^{2} / \mathrm{m} / \mathrm{s}$ [21] while in a tunnel built in Japan, $A=2 * 10^{-9} \mu \mathrm{m}^{2} / \mathrm{m} / \mathrm{s}$ was observed [22].

Recent measurements at SLAC have shown that the value of A can depend on changes in atmospheric pressure acting on the ground [18]. These observations are explained by a variation of the ground properties along the linac. This variation can be due to changes in the Young's modulus $E$, changes in the topology of the surface, or changes in the characteristic depth of the softer surface layers. In this case the atmosphere-driven contribution to A scales as $1 / E^{2}$ and, therefore, depends strongly on geology. This may be partly responsible for the large variation of A observed at different sites.

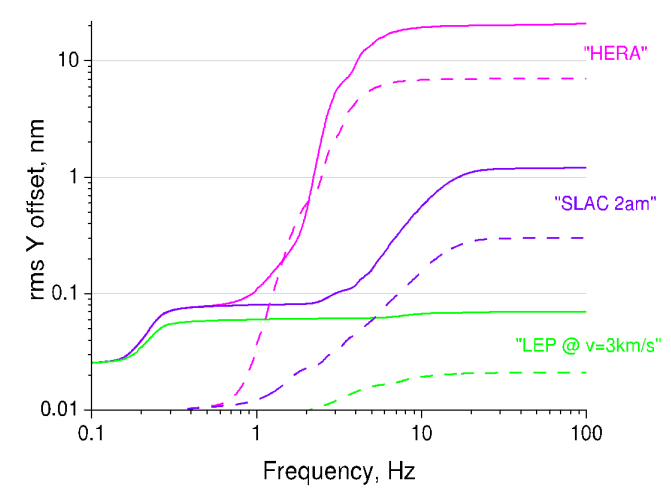

Figure 6: Integrated spectral contribution to the RMS equilibrium IP beam offset for the NLC Final Focus with final doublets supports at $\pm 8 \mathrm{~m}$ for different models of ground motion. Dashed curves correspond to the case where the relative motion of the FDs is eliminated [23].

Very slow ground motion can also be systematic in time over periods of months to years. Such motion has been observed at SLAC, CERN as well as other places [24]. In some cases such motion can be described by a simple rule for its RMS relative misalignment [14]: $\Delta \mathrm{X}^{2}=$ $\mathrm{A}_{\mathrm{S}} \mathrm{T}^{2} \mathrm{~L}$ with another site-specific coefficient $\mathrm{A}_{\mathrm{S}}$. Immediately after construction of SLAC, it appears that the systematic motion dominated on a time scale greater than a day however it is significantly reduced nowadays. It is important to note that earlier studies of the ATL law suggested higher values of the parameter A. One reason for this, especially for the SLAC data, was a misinterpretation of year-to-year systematic motion as diffusive motion. As seen from Fig.4, this confusion resulted in the overestimation of A by more than two orders of magnitude. Studies to investigate slow ground motion in more detail are in progress at many different laboratories [25].
Modeling the ground motion is an important step towards accurately characterizing the influence of ground motion on a linear collider. The model should include an understanding of the temporal and spatial properties of the motion and of the driving mechanisms. In most cases, an adequate representation for such a model consists of the 2-D power spectrum $\mathrm{P}(\omega, \mathrm{k})$ based on measured spectra of absolute motion and correlation. Recently, several models representing different conditions have been developed and used to evaluate NLC performance. The models are referred to as 'SLAC site', 'HERA site' and 'LEP tunnel' [14].

As an example of how such models can be used, a comparison of the performance of the NLC Final Focus for different ground motion models is shown in Figure 6. One can see that a site located in a highly populated area without proper vibration-sensitive engineering would present significant difficulties for a linear collider with the parameters considered. Stabilization of more components than just the final doublet would be necessary. A site with noise similar to the 'SLAC site model' would certainly be suitable, while the 'LEP tunnel model' would be suitable even for much more ambitious beam parameters.

\section{NLC STABILIZATION}

For the NLC, the ground motion and vibration issues have been systematically addressed by proper design, careful consideration of sites and geology, and by development of appropriate stabilization methods.

Feedback systems are an essential component of NLC. For the SLAC ground motion model, simulations show that the linac orbit feedback can suppress the beam motion at the end of the linac to less than $4 \%$ of the beam size, while it can be as much as $30 \%$ of the beam size without feedback [26].

For diffusive motion with a value of $\mathrm{A}=5 * 10^{-7}$ $\mu \mathrm{m}^{2} / \mathrm{m} / \mathrm{s}$, similar to observations at SLAC in the SLC and FFTB tunnels $[27,28,18]$, the NLC linac feedback can maintain the orbit for several hours before steering (a nondisruptive procedure) is required to restore the smoothness of the beam line [26]. For the Beam Delivery System, feedback to correct the trajectory and to optimize the luminosity together with first order aberration knobs can maintain optimal performance of the system for nearly one year [29]. Eventually, a beam-based quadrupole alignment must be reapplied.

The site location is clearly an important issue for the NLC design. An ideal site should have little external cultural noise, now and in the future. Solid rock is the preferred surrounding media since fast motion will be better correlated and slow motion will be reduced. In real life, proximity to an existing major laboratory would also be a great advantage. This proximity would not necessarily mean compromising the other requirements since a deep tunnel version of the NLC, considered in 
both Illinois and California, could have good geology and low noise and still be located near an existing laboratory.

Of course, a good site alone is not sufficient. Noise generated by the linear collider equipment itself and by conventional facilities equipment must be appropriately controlled and minimized (by design and by further passive or active damping). This should allow the tolerances to be met for all of the NLC focusing and accelerating elements, except for the final doublet (FD), without any additional active stabilization or correction.

Although the NLC detector must be designed to minimize vibrations, it is unlikely that the FD mounted on the detector can meet the position stability tolerances without additional active measures. Several methods are being developed to provide the necessary relative stability between the FDs. These include position stabilization via feedback, and correction of the magnetic center position with dipole coils via feedforward. Both methods would rely on either inertial measurements of the motion by seismometers or on optical interferometric measurements of their position with respect to each other or to stable ground under the detector (with an 'Optical anchor'). A prototype optical anchor has demonstrated a resolution of about $0.2 \mathrm{~nm}$ [30] that would be sufficient for measuring the position of the FD. It is now being used for stabilization tests [31].

An attempt to stabilize the position of a quadrupole was made at DESY as part of the S-Band linear collider project [32]. A single seismometer and a single piezomover were used to stabilize the effective position of the quadrupole center. A reduction of RMS motion by a factor of 3 was achieved (from $100 \mathrm{~nm}$ to about $30 \mathrm{~nm}$ for frequencies higher than a few $\mathrm{Hz}$ ). Another attempt at inertial stabilization was performed at SLAC using three commercial STACIS insulation stands [33] to stabilize a $1500 \mathrm{~kg}$ PEP-II quadrupole [34,35]. In this test, the floor motion was reduced by about a factor of 20 (from $40 \mathrm{~nm}$ to $2 \mathrm{~nm}$ for $\mathrm{f}>2 \mathrm{~Hz}$ ). However, additional slow noise of the order of $200 \mathrm{~nm}$ was introduced and the performance in the horizontal plane was not as satisfactory. These first examples of inertial stabilization (or inertial sensing for feedforward) do not yet satisfy NLC requirements. In a real collider, the system must detect and minimize motion of two extended and separated FDs without disturbing the correlation with the rest of machine; it must work in an external magnetic field, be compact and reliable. Ongoing work $[31,36,37]$ will address these issues. At SLAC, preliminary tests of the recently developed inertial stabilization system with digital feedback in 6D resulted in more than an order of magnitude reduction of motion of a test object [36].

In addition to these methods of stabilization, a fast correction within the bunch train is being developed for NLC (similar to TESLA). The intratrain feedback uses a position monitor (BPM) near the IP to detect the offset of the first bunches of the train. The signal is the beam-beam deflection due to the relative offset of the beams and fast kickers are then used to correct the rest of the bunch train. Recent evaluation indicates that such a system is technically feasible with available components and could provide efficient capture of beams with several sigma offset [38]. For example, with a $10 \mathrm{~nm}$ beam offset essentially full luminosity would be restored after roughly $15 \%$ of the bunch train. This would significantly reduce the requirements on incoming beam jitter and on stabilization of the FD.

\section{CONCLUSION}

Ground motion and vibration are important issues for any future collider. For the NLC, these issues have been extensively studied. Stabilization solutions have been found that should provide the required stability of the NLC beam quality and luminosity.

This paper summarizes results obtained by many people from laboratories throughout the world. The author would like to acknowledge all of their contributions.

\section{REFERENCES}

[1] NLC ZDR Design Group, SLAC Report-474 (1996).

[2] 2001 Report on the NLC, SLAC-R-571, June 2001.

[3] JLC projects, http://www-jlc.kek.jp/

[4] TESLA TDR, March 2001.

[5] VLHC project, http://vlhc.org/

[6] G.Guignard, editor, CERN Report 2000-008.

[7] B.Baklakov et al., Ph.Rev. STAB, 1, 031001 (1998).

[8] G.Stupakov in SLAC WP-18, 2001 and refs therein.

[9] M.Blaskiewicz, et al., SLAC-PUB-8800, 2001.

[10] V.M. Juravlev et al. HU-SEFT R 1995-01.

[11] C.Montag, V.Shiltsev, et al., DESY HERA 95-06, 1995.

[12] A.Sery,O.Napoly, PRE 535323 (1996) + refs therein.

[13] R.Fenn, et al., SLAC LCC-Note-36, 2000.

[14] A.Seryi, T.Raubenheimer, SLAC-PUB-8595, 2000.

[15] V.M. Juravlev et al. CERN-SL-93-53.

[16] B. Baklakov, et al. Tech. Phys. 38, 894 (1993).

[17] LIGO project, http://www.ligo.caltech.edu/

[18] A.Seryi, SLAC-PUB-8597, 2000.

[19] F.Asiri, SLAC, R.Schofield, LIGO, private communic.

[20] V. Shiltsev, in Proc. IWAA95, KEK-95-12, 1995.

[21] R.Brinkmann, J.Rossbach, NIM Ph.Res. A 350, 8 (1994).

[22] S.Takeda, et al., KEK 99-135, in Proc. IWAA 1999.

[23] A.Seryi,et al, SLAC-PUB-8596, 2000.

[24] R.Pitthan, SLAC-PUB-7043, SLAC-PUB-8286, 1995, 99.

[25] V.Shiltsev et al., these Proceedings.

[26] L.Hendrickson et al., these Proceedings.

[27] C.Adolphsen, G.Bowden, G.Mazaheri, in Proc. of LC97.

[28] R.Assmann, C.Salsberg, C.Montag, SLAC-PUB-7303.

[29] P.Raimondi, et al., in these Proceedings.

[30] M.Woods, in Proc of LC97, Zvenigorod, 1997, p.1591.

[31] T.Mattison, in SLAC-WP-18, 2001, p 567.

[32] C.Montag, DESY-97-030, Feb. 1997.

[33] TMC, STACIS, http://www.techmfg.com/

[34] G.Bowden, G.Mazaheri, NLC ME Note 7-96, June 1996.

[35] G.Bowden et al., in SLAC-WP-18, 2001, p.527.

[36] J.Frisch et al., in these Proceedings.

[37] CLIC stability studies, http://clic-stability.web.cern.ch/

[38] S.Smith, LCC Note 0056, March 2001, SLAC. 